\title{
Cytotoxicity of New 5-Phenyl-4,5-dihydro-1,3,4-thiadiazole Analogues
}

\author{
Mohammad Sayed Alam, ${ }^{a, c}$ Lijun Liu, ${ }^{b}$ and Dong Ung LeE* ${ }^{*} a$ \\ ${ }^{a}$ Division of Bioscience, Dongguk University; Gyeongju 780-714, Republic of Korea: ${ }^{b}$ College of Chemistry and \\ Chemical Engineering, Ningxia University; Yinchuan 750021, China: and ' Department of Chemistry, Jagannath \\ Univeristy; Dhaka 1100, Bangladesh. \\ Received June 14, 2011; accepted August 22, 2011; published online August 25, 2011
}

A series of 5-phenyl-4,5-dihydro-1,3,4-thiadiazoles were synthesized and their cytotoxicity was examined against four human cancer cell lines, e.g. lung cancer (A549), ovarian cancer (SK-OV-3), skin cancer (SK-MEL2), and colon cancer (HCT15). The title compounds were synthesized by condensation of thiosemicarbazide with substituted benzaldehydes, followed by cyclization with acetic anhydrides in good yields. Most of the compounds exhibited significant suppressive activity against the growth of all of the cancer cell lines. The 4-hydroxy analogue of 5-phenyl-4,5-dihydro-1,3,4-thiadiazole $(2 \mathrm{~h})$ was most active in the inhibition of growth of the SK-MEL-2 cell line, with an $\mathrm{IC}_{50}$ value of $4.27 \mu \mathrm{g} / \mathrm{ml}$; followed by compound $2 \mathrm{a}\left(\mathrm{IC}_{50} 5.16 \mu \mathrm{g} / \mathrm{ml}\right)$. The compounds $2 \mathrm{j}$, $2 \mathrm{~h}$, and $2 \mathrm{~b}$, bearing 3-methoxy-4-hydroxy-, 4-hydroxy- and 4-methyl substituents in the C-5 phenyl ring respectively, exhibited the highest activity against the SK-OV-3 $\left(\mathrm{IC}_{50} 7.35 \mu \mathrm{g} / \mathrm{ml}\right), \mathrm{HCT15}\left(\mathrm{IC}_{50} 8.25 \mu \mathrm{g} / \mathrm{ml}\right)$ and $\mathrm{A5}_{49}\left(\mathrm{IC}_{50}\right.$ $9.40 \mu \mathrm{g} / \mathrm{ml}$ ) cell lines, respectively. A structure-activity relationship study revealed that an optimal electron density on the C-5 phenyl ring of 1,3,4-thiadiazoles is crucial for their cytotoxic activity against the human cancer cell lines used in the present study.

Key words 5-phenyl-4,5-dihydro-1,3,4-thiadiazole; cytotoxicity; human cancer cell line

Phenyl-1,3,4-thiadiazoles are a class of small molecules that have received much interest in the fields of chemistry and biology due to their broad spectrum of activity. The 1,3,4-thiadiazole scaffold is an interesting building block that has been used to synthesize a variety of useful bioactive compounds. There is a progressive body of studies on the synthesis of 1,3,4-thiadiazole analogues and their wide range of pharmacological actions in the literature. Phenyl-1,3,4thiadiazole derivatives have been reported to be antimicrobial, ${ }^{1)}$ anticancer, ${ }^{2)}$ anti-tubercular, ${ }^{3)}$ anti-convulsant, ${ }^{4)}$ antiinflammatory, ${ }^{5)}$ analgesic, ${ }^{5)}$ anti-anxiety, anti-depressant ${ }^{6)}$ and anti-viral ${ }^{7)}$ agents.

Cancer is one of the major causes of death globally. Many anticancer agents exert their effects through the destruction of rapidly dividing cells, and these cytotoxic agents remain the primary resource in cancer chemotherapy, despite advances in understanding of the cell cycle that may help develop more selective chemotherapeutic agents that target only cancer cells. During the last few decades, anticancer therapy has progressed significantly, but the management of malignancies in humans still constitutes a major concern for contemporary medicine. Phenyl-1,3,4-thiadiazole and its derivatives are well known, broad spectrum anticancer agents $^{8-10)}$ that have been proven in in vivo conditions. ${ }^{11,12)}$

As a part of our continuing search for novel biologically active molecules, we have designed and synthesized 5phenyl-4,5-dihydro-1,3,4-thiadiazole analogues $(\mathbf{2 a}-\mathbf{j})$ and evaluated their anticancer properties. The compounds $\mathbf{2} \mathbf{a}-\mathbf{j}$ were synthesized, by modification of a known method, ${ }^{13)}$ from their corresponding thiosemicarbazone analogues $(\mathbf{1 a}-$ j). Furthermore, we investigated the in vitro cytotoxic activities of these new compounds against four culture cell lines: A549 (human lung cancer), SK-OV-3 (human ovarian cancer), SK-MEL-2 (human skin cancer), and HCT15 (human colon cancer).

Synthesis of 5-Phenyl-4,5-dihydro-1,3,4-thiadiazoles Synthesis of the 5-phenyl-4,5-dihydro-1,3,4-thiadiazole ana- logues $(\mathbf{2} \mathbf{a}-\mathbf{j})$ was achieved following a convenient two-step procedure; starting from commercially available starting materials as outlined in Chart 1. The intermediate arylidenethiosemicarbazone analogues $(\mathbf{1} \mathbf{a}-\mathbf{j})$ were synthesized by the condensation reaction of thiosemicarbazide and substitution of benzaldehyde in ethanol, with excellent yields (80-98\%). The structures of compounds $\mathbf{1 a}-\mathbf{j}$ were confirmed by IR and ${ }^{1} \mathrm{H}-\mathrm{NMR}$ spectral data. In the IR spectrum of the arylidenethiosemicarbazones $(\mathbf{1} \mathbf{a}-\mathbf{j})$, the $-\mathrm{NH}_{2}$ and -NH- groups showed an absorption band at around the $3490-3373 \mathrm{~cm}^{-1}$ and $3363-3239 \mathrm{~cm}^{-1}$ regions, respectively. In the ${ }^{1} \mathrm{H}-\mathrm{NMR}$ spectra of compounds $\mathbf{1 a}-\mathbf{k}$, two amino $\left(-\mathrm{NH}_{2}\right)$ protons appeared as two singlets at $7.67-$ 8.26 and $7.81-8.38 \mathrm{ppm}$ due to en-thiol tautomerism, while these protons disappeared in compounds $\mathbf{2 a - j}$. The $=\mathrm{N}-\mathrm{NH}-$ and $-\mathrm{CH}=\mathrm{N}-$ protons were observed as singlet at $11.17-11.71$ and $7.93-8.41 \mathrm{ppm}$, respectively, equivalent to one proton each. The aromatic proton signals appeared at $6.67-8.63 \mathrm{ppm}$, either as multiplets or doublets, according to the substitution pattern on the phenyl ring.

The cyclization of arylidenethiosemicarbazones $(\mathbf{1} \mathbf{a}-\mathbf{j})$, acheived by refluxing with acetic anhydrides in ethanol, yielded 5-phenyl-4,5-dihydro-1,3,4-thiadiazole $(\mathbf{2 a - j})$ analogues in good yields $(67-80 \%)$. All the synthesized 5phenyl-4,5-dihydro-1,3,4-thiadiazoles were characterized by IR, ${ }^{1} \mathrm{H}-\mathrm{NMR}$, and electron ionization (EI)-MS spectral data together with elemental analysis. In the IR spectrum of compounds $2 \mathbf{a}-\mathbf{j}$, the characteristic $\mathrm{N}-\mathrm{H}$ and $>\mathrm{C}=\mathrm{O}$ stretching absorption bands appeared at the $3222-3203 \mathrm{~cm}^{-1}$ and $1713-1631 \mathrm{~cm}^{-1}$ regions, respectively. The ${ }^{1} \mathrm{H}-\mathrm{NMR}$ of compounds $\mathbf{2 a}-\mathbf{j}$ showed a characteristic singlet for the C-5 proton at $6.75-6.89 \mathrm{ppm}$, with an upfield shift of 1.18 $1.52 \mathrm{ppm}$ from that of the imino proton $(-\mathrm{CH}=\mathrm{N}-)$ of the open-chain thiosemicarbazones $(\mathbf{1} \mathbf{a}-\mathbf{j})$, which was in good agreement with other reported chemical shifts of 1,3,4-thiadiazole analogues. ${ }^{14,15)}$ Two acetyl groups appeared as two singlets at $2.01-2.12$ and $2.23-2.30 \mathrm{ppm}$. The amide pro- 
<smiles>[R]c1ccc(C=O)cc1[R]</smiles><smiles>[3H][13CH]</smiles><smiles>[R]c1ccc(/C=N\NC(N)=S)cc1[R]</smiles>

b<smiles>[R]c1ccc(C2SC(NC=O)=NN2C(C)=O)cc1[R]</smiles>

Chart 1. Preparation of New 1,3,4-Thiadiazole Analogues 2a-j

(a) EtOH- $\mathrm{H}_{2} \mathrm{O}$, reflux; (b) $\left(\mathrm{CH}_{3} \mathrm{CO}\right)_{2} \mathrm{O}$, EtOH, reflux.

ton (-NHCO-) of compounds $\mathbf{2} \mathbf{a}-\mathbf{j}$ was observed as a singlet at $11.63-11.89 \mathrm{ppm}$. The phenyl protons were assigned in the usual way, according to their substitution pattern. EIMS spectra of $\mathbf{2} \mathbf{a}-\mathbf{j}$ showed a molecular ion peak with intensities from $85-100 \%$.

Anticancer Activity The anticancer activity of compounds $\mathbf{2} \mathbf{a}-\mathbf{j}$ was evaluated by an in vitro assay performed on four human cancer cell lines: lung cancer (A549), ovarian cancer (SK-OV-3), skin cancer (SK-MEL-2), and colon cancer (HCT15). The activity was evaluated by measuring the inhibition of the net growth of cells, measured as a percent of the control samples, after incubation for $48 \mathrm{~h}$ with the test samples, using the SRB (sulforhodamine-B) method. All activities were compared with cisplatin as a positive control. The activity results presented in Table 1 report the cytotoxic effects of 5-phenyl-4,5-dihydro-1,3,4-thiadiazole $(\mathbf{2 a - j})$ analogues against the various cancer cell lines. Among the tested compounds $\mathbf{2} \mathbf{a}, \mathbf{b}, \mathbf{h}$ and $\mathbf{j}$ showed significant cytotoxic effects against all the cancer cell lines. Compound $\mathbf{2} \mathbf{h}$ exhibited the highest activity $\left(\mathrm{IC}_{50} 4.27 \mu \mathrm{g} / \mathrm{ml}\right)$ and selectivity, followed by $\mathbf{2 a}\left(\mathrm{IC}_{50} 5.16 \mu \mathrm{g} / \mathrm{ml}\right)$, against the skin cancer (SKMEL-2) cell line. While compound $\mathbf{2} \mathbf{j}$ exhibited the highest cytotoxic activity $\left(\mathrm{IC}_{50} 7.35 \mu \mathrm{g} / \mathrm{ml}\right)$ and selectivity against the ovarian cancer (SK-OV-3) cell line, followed by $\mathbf{2 h}\left(\mathrm{IC}_{50}\right.$ $10.14 \mu \mathrm{g} / \mathrm{ml}$ ). Compound 2b exhibited significant potency against the lung cancer (A549) cell line, with an $\mathrm{IC}_{50}$ value of $9.40 \mu \mathrm{g} / \mathrm{ml}$; followed by compounds $\mathbf{2 a}\left(\mathrm{IC}_{50} 14.27 \mu \mathrm{g} / \mathrm{ml}\right)$ and $\mathbf{2 h}\left(\mathrm{IC}_{50} 15.58 \mu \mathrm{g} / \mathrm{ml}\right)$. Compound $\mathbf{2} \mathbf{h}$ exhibited significant potency against the colon cancer (HCT15) cell line, with an $\mathrm{IC}_{50}$ value of 8.25 ; followed by compounds $\mathbf{2 a}, \mathbf{j}$ and $\mathbf{b}$, at $10.25,10.83$ and $12.24 \mu \mathrm{g} / \mathrm{ml}$, respectively.

The structure-activity relationship study revealed that the nature of the substituted group on the phenyl ring of 5-
Table 1. In Vitro Cytotoxicity Data of the 5-Phenyl-4,5-dihydro-1,3,4-thiadiazole Analogues $(\mathbf{2} \mathbf{a}-\mathbf{j})$ against Selected Human Cancer Cell Lines<smiles>[R]c1ccc(C2SC(NC(C)=O)=NN2C(C)=O)cc1[R]</smiles>

\begin{tabular}{cccrcrr}
\hline \hline & & & \multicolumn{5}{c}{$\mathrm{IC}_{50}(\mu \mathrm{g} / \mathrm{ml})^{a)}$} \\
\cline { 4 - 7 } Compd. & $\mathrm{R}_{1}$ & $\mathrm{R}_{2}$ & $\mathrm{~A} 549$ & $\mathrm{SK}-\mathrm{OV}-3$ & $\mathrm{SK}-\mathrm{SEL}-2$ & $\mathrm{HCT} 15$ \\
& & & & & & \\
\hline $\mathbf{2 a}$ & $\mathrm{H}$ & $\mathrm{H}$ & 14.27 & 13.22 & 5.16 & 10.25 \\
$\mathbf{2 b}$ & $\mathrm{Me}$ & $\mathrm{H}$ & 9.40 & 13.39 & 11.05 & 12.24 \\
$\mathbf{2 c}$ & $\mathrm{NO}_{2}$ & $\mathrm{H}$ & 102.11 & 108.67 & 105.25 & 98.71 \\
$\mathbf{2 d}$ & $\mathrm{H}$ & $\mathrm{NO}_{2}$ & 135.25 & 175.58 & 139.21 & 140.65 \\
$\mathbf{2 e}$ & $\mathrm{NMe}_{2}$ & $\mathrm{H}$ & 144.85 & 187.62 & 179.56 & 135.15 \\
$\mathbf{2 f}$ & $\mathrm{Cl}$ & $\mathrm{H}$ & 72.61 & 78.52 & 53.33 & 60.27 \\
$\mathbf{2 g}$ & $\mathrm{Br}$ & $\mathrm{H}$ & 60.29 & 21.76 & 28.73 & 74.82 \\
$\mathbf{2 h}$ & $\mathrm{OH}$ & $\mathrm{H}$ & 15.58 & 10.14 & 4.27 & 8.25 \\
$\mathbf{2 i}$ & $\mathrm{OMe}$ & $\mathrm{H}$ & 180.12 & 188.75 & 168.16 & 137.86 \\
$\mathbf{2 j}$ & $\mathrm{OH}$ & $\mathrm{OMe}$ & 28.16 & 7.35 & 29.76 & 10.83 \\
& $\mathrm{Cisplatin}$ & & 1.4 & 0.9 & 0.8 & 2.2 \\
\hline
\end{tabular}

a) $\mathrm{IC}_{50}$ values were obtained using a dose response curve by nonlinear regression using a curve fitting program, OriginPro 7.5.

phenyl-4,5-dihydro-1,3,4-thiadiazole plays an important role in the cytotoxic activity of the compound. Compound 2a, which possessed an unsubstituted phenyl ring, was 2-3 times more cytotoxic against SK-MEL-2 cell lines $\left(\mathrm{IC}_{50}\right.$ $5.16 \mu \mathrm{g} / \mathrm{ml}$ ) than against the A549, SK-OV-3 and HCT15 cell lines. Introduction of a methyl group, a weak electron-donating group, at the para-position on the phenyl ring resulted in compound $\mathbf{2 b}$, which exhibited greater activity against A549 cell lines than against SK-MEL-2 and HCT15, but was simi- 
lar to that against the ovarian cancer (SK-OV-3) cell lines. Introduction of a hydroxy group, which has more electron-donating capacity than a methyl group, yielded compound $\mathbf{2 h}$, which had enhanced activity against all the cell lines, except A549, compared with that of $\mathbf{2 a}$. Increasing the electron-donating capacity of the hydroxy group, by replacing it with methoxy group (compound $\mathbf{2 i}$ ), led to a substantial loss in activity against all cell lines. N,N-dimethylamino group, a stronger electron-donating group than a hydroxy or a methoxy group, at the para-position of the phenyl ring (compound 2e) also markedly reduced activity against all cell lines. Bulkiness of the substituents influenced cytotoxicity; more bulky group at the para-position on the aromatic ring than hydroxy group (2h), such as methoxy (2i), N,N-dimethylamino (2e) and nitro group (2c), reduced a potency drastically. In another point of view, the proton donating ability of a hydroxyl group of compounds $\mathbf{2} \mathbf{h}$ and $\mathbf{j}$ may also play a crucial role for the cytotoxicity. Compounds having a poor proton donating property $(\mathbf{2} \mathbf{c}, \mathbf{e}, \mathbf{i})$ exhibited much weaker activity than compounds $\mathbf{2} \mathbf{h}$ and $\mathbf{j}$ which possess a good proton donating ability. Therefore, a phenyl-substituted thiadiazole moiety would be suggested as a target site of the molecule for the strong cytotoxicity of 1,3,4-thiadiazle analogs. The proton donating ability has been discussed to be critical for the antimicrobial activity of trityl derivatives. ${ }^{16)}$ Introduction of a methoxy group at the meta-position of compound $\mathbf{2 h}$ yielding compound $\mathbf{2} \mathbf{j}$, resulted in improved activity against the SK-OV-3 cell lines and decreased activity against the other three. Introducing a nitro group, a strong electron-withdrawing group, at the para- or meta-position on the phenyl ring (compounds $\mathbf{2 c}$, $\mathbf{d}$, respectively) resulted in reduced activity compared to that of compound $\mathbf{2 a}$, but exhibited similar activities to compounds $\mathbf{2 e}$ and i. Compounds with halogen substituents (2f, $\mathbf{g})$, which have a weak electron-withdrawing capacity but also have an electron donating ability due to resonance, displayed improved cytotoxicity against all cell lines compared to compounds $\mathbf{2 c}$ and $\mathbf{d}$. The bromine substituted compound $\mathbf{2 g}$, showed increased activity against the SK-OV-3 and SK-MEL-2 cell lines compared to that of the chlorine substituted compound $\mathbf{2 f}$; while both compounds (2f, g) had similar activity against the A549 and HCT15 cell lines.

The above structure-activity relationships led us to hypothesize that an optimum electron density, a substituent bulkiness or a proton donating ability in the C-5 phenyl ring of 1,3,4-thiadiazoles may be closely related for maximum cytotoxic activity against the cell lines used in the present study. Further structure-activity studies with versatile analogs are needed to clearly elucidate the role of above points on the cytotoxicity of 1,3,4-thiadiazoles and identify their molecular targets.

\section{Experimental}

General Melting points were determined on an X-5 melting point apparatus (Yuxiagyiqi, Gongyi City Yuxiang Instruments Co., Ltd., China) and are uncorrected. IR spectra were obtained with an FTIR-8430S (Shimadzu, Japan) using $\mathrm{KBr}$ discs. NMR spectra were recorded with an AM-400MH (Bruker, U.S.A.) using DMSO- $d_{6}$ with tetramethylsilane as an internal standard. Mass spectra were measured with an HP-5988 spectrometer (EI, $70 \mathrm{eV})$. Elemental analyses $(\mathrm{C}, \mathrm{H}, \mathrm{N})$ were performed by means of a PerkinElmer 2400 II CHN elemental analyzer.

Preparation of Thiosemicarbazone Analogues 1a-j The synthesis of thiosemicarbazones was carried out according to the previously described method. ${ }^{17)}$ Briefly, an ethanolic solution of substituted benzaldehydes $(1 \mathrm{mmol})$ was added slowly to a stirred solution of thiosemicarbazide $(1 \mathrm{mmol})$ in an ethanol-water mixture, and refluxed for $10-20 \mathrm{~min}$. After cooling the reaction mixture to an ambient temperature, the mixture was filtered to give a solid crude product, which was crystallized from ethanol to provide the pure compounds $1 \mathbf{a}-\mathbf{j}$ with a yield of $80-98 \%$.

2-Benzylidenehydrazinecarbothioamide 1a: Yield $98 \%$. mp $164-165^{\circ} \mathrm{C}$. ${ }^{1} \mathrm{H}-\mathrm{NMR} \delta$ : 7.35-7.52 (m, 3H, Ar-H), 7.87-8.00 (m, 2H, Ar-H), 7.74 and $7.81\left(2 \times \mathrm{s}, 2 \mathrm{H},-\mathrm{NH}_{2}\right), 8.17(\mathrm{~s}, 1 \mathrm{H}, \mathrm{CH}), 11.40(\mathrm{~s}, 1 \mathrm{H},-\mathrm{NH}-)$. IR $(\mathrm{KBr})$ $\mathrm{cm}^{-1}: 3422\left(\mathrm{NH}_{2}\right), 3251(\mathrm{NH}), 1590(\mathrm{C}=\mathrm{N}), 1298(\mathrm{C}=\mathrm{S})$.

2-(4-Methylbenzylidene)hydrazinecarbothioamide 1b: Yield $80 \%$. mp 175-176 ${ }^{\circ} \mathrm{C} .{ }^{1} \mathrm{H}-\mathrm{NMR} \delta: 2.42\left(\mathrm{~s}, 3 \mathrm{H},-\mathrm{CH}_{3}\right), 6.96(\mathrm{~d}, 2 \mathrm{H}, J=8.2 \mathrm{~Hz}, \mathrm{Ar}-$ $\mathrm{H}), 7.76(\mathrm{~d}, 2 \mathrm{H}, J=8.2 \mathrm{~Hz}, \mathrm{Ar}-\mathrm{H}), 7.92$ and $8.12\left(2 \times \mathrm{s}, 2 \mathrm{H},-\mathrm{NH}_{2}\right), 8.03(\mathrm{~s}$, $1 \mathrm{H}, \mathrm{CH}), 11.33$ (s, 1H, -NH-). IR (KBr) cm ${ }^{-1}: 3390\left(\mathrm{NH}_{2}\right), 3270(\mathrm{NH})$, $1610(\mathrm{C}=\mathrm{N}), 1260(\mathrm{C}=\mathrm{S})$.

2-(4-Nitrobenzylidene)hydrazinecarbothioamide 1c: Yield 98\%. $\mathrm{mp}$ $230-231{ }^{\circ} \mathrm{C} .{ }^{1} \mathrm{H}-\mathrm{NMR} \delta: 8.07(\mathrm{~d}, 2 \mathrm{H}, J=8.2 \mathrm{~Hz}$, Ar- $\mathrm{H}), 8.22(\mathrm{~d}, 2 \mathrm{H}$, $J=8.2 \mathrm{~Hz}, \mathrm{Ar}-\mathrm{H}), 8.26$ and $8.38\left(2 \times \mathrm{s}, 2 \mathrm{H},-\mathrm{NH}_{2}\right), 8.41(\mathrm{~s}, 1 \mathrm{H}, \mathrm{CH}), 11.71$ (s, $1 \mathrm{H},-\mathrm{NH}-)$. IR (KBr) cm ${ }^{-1}: 3490\left(\mathrm{NH}_{2}\right), 3363(\mathrm{NH}), 1589(\mathrm{C}=\mathrm{N}), 1247$ $(\mathrm{C}=\mathrm{S})$.

2-(3-Nitrobenzylidene)hydrazinecarbothioamide 1d: Yield 98\%. $\mathrm{mp}$ 215-216 ${ }^{\circ} \mathrm{C} .{ }^{1} \mathrm{H}-\mathrm{NMR} \delta: 7.64-7.81(\mathrm{~m}, 2 \mathrm{H}, \mathrm{Ar}-\mathrm{H}), 8.42-8.63(\mathrm{~m}, 2 \mathrm{H}$, Ar-H), 7.67 and $8.18\left(2 \times \mathrm{s}, 2 \mathrm{H},-\mathrm{NH}_{2}\right), 8.19(\mathrm{~s}, 1 \mathrm{H}, \mathrm{CH}), 11.60(\mathrm{~s}, 1 \mathrm{H}$, $-\mathrm{NH}-)$. IR (KBr) cm ${ }^{-1}: 3393\left(\mathrm{NH}_{2}\right), 3239(\mathrm{NH}), 1603(\mathrm{C}=\mathrm{N}), 1299(\mathrm{C}=\mathrm{S})$.

2-(4-(Dimethylamino)benzylidene)hydrazinecarbothioamide 1e: Yield $86 \%$. mp $193-194{ }^{\circ} \mathrm{C} .{ }^{1} \mathrm{H}-\mathrm{NMR} \delta: 2.94\left(\mathrm{~s}, 6 \mathrm{H}, \mathrm{CH}_{3}\right), 6.67(\mathrm{~d}, 2 \mathrm{H}$, $J=8.2 \mathrm{~Hz}, \mathrm{Ar}-\mathrm{H}), 7.55(\mathrm{~d}, 2 \mathrm{H}, J=8.2 \mathrm{~Hz}, \mathrm{Ar}-\mathrm{H}), 7.75$ and $7.91(2 \times \mathrm{s}, 2 \mathrm{H}$, $\left.-\mathrm{NH}_{2}\right), 7.99$ (s, 1H, CH), 11.17 (s, 1H, NH). IR $(\mathrm{KBr}) \mathrm{cm}^{-1}: 3373\left(\mathrm{NH}_{2}\right)$, $3330(\mathrm{NH}), 1600(\mathrm{C}=\mathrm{N}), 1269(\mathrm{C}=\mathrm{S})$.

2-(4-Chlorobenzylidene)hydrazinecarbothioamide 1f: Yield $89 \%$. mp 209-211 ${ }^{\circ} \mathrm{C} .{ }^{1} \mathrm{H}-\mathrm{NMR} \delta: 7.43(\mathrm{~d}, 2 \mathrm{H}, J=8.2 \mathrm{~Hz}$, Ar-H), $7.84(\mathrm{~d}, 2 \mathrm{H}$, $J=8.2 \mathrm{~Hz}, \mathrm{Ar}-\mathrm{H}), 8.00$ and $8.02\left(2 \times \mathrm{s}, 2 \mathrm{H},-\mathrm{NH}_{2}\right), 8.23(\mathrm{~s}, 1 \mathrm{H}, \mathrm{CH}), 11.47$ (s, $1 \mathrm{H},-\mathrm{NH}-)$. IR $(\mathrm{KBr}) \mathrm{cm}^{-1}: 3436\left(\mathrm{NH}_{2}\right), 3278(\mathrm{NH}), 3105(\mathrm{C}-\mathrm{H}), 1600$ $(\mathrm{C}=\mathrm{N}), 1282(\mathrm{C}=\mathrm{S})$.

2-(4-Bromobenzylidene)hydrazinecarbothioamide 1g: Yield 91\%. $\mathrm{mp}$ 219-220 ${ }^{\circ} \mathrm{C}$. ${ }^{1} \mathrm{H}-\mathrm{NMR} \delta$ : $7.43(\mathrm{~d}, 2 \mathrm{H}, J=8.1 \mathrm{~Hz}$, Ar-H), $7.84(\mathrm{~d}, 2 \mathrm{H}$, $J=8.1 \mathrm{~Hz}, \mathrm{Ar}-\mathrm{H}), 8.01$ and $8.07\left(2 \times \mathrm{s}, 2 \mathrm{H},-\mathrm{NH}_{2}\right), 8.23(\mathrm{~s}, 1 \mathrm{H}, \mathrm{CH}), 11.47$ (s, $1 \mathrm{H},-\mathrm{NH}-)$. IR $(\mathrm{KBr}) \mathrm{cm}^{-1}: 3431\left(\mathrm{NH}_{2}\right), 3278(\mathrm{NH}), 1609(\mathrm{C}=\mathrm{N}), 1279$ $(\mathrm{C}=\mathrm{S})$.

2-(4-Hydroxybenzylidene)hydrazinecarbothioamide $\mathbf{1 h}$ : Yield 92\%. mp $217-218^{\circ} \mathrm{C} .{ }^{1} \mathrm{H}-\mathrm{NMR} \delta: 6.73(\mathrm{~d}, 2 \mathrm{H}, J=8.2 \mathrm{~Hz}$, Ar- $\mathrm{H}), 7.60(\mathrm{~d}, 2 \mathrm{H}$, $J=8.2 \mathrm{~Hz}, \mathrm{Ar}-\mathrm{H}), 7.81$ and $7.91\left(2 \times \mathrm{s}, 2 \mathrm{H},-\mathrm{NH}_{2}\right), 8.04(\mathrm{~s}, 1 \mathrm{H}, \mathrm{CH}), 9.84(\mathrm{~s}$ $1 \mathrm{H}, \mathrm{OH}), 11.22(\mathrm{~s}, 1 \mathrm{H},-\mathrm{NH}-)$. IR $(\mathrm{KBr}) \mathrm{cm}^{-1}: 3467\left(\mathrm{NH}_{2}\right), 3359(\mathrm{NH})$, $1608(\mathrm{C}=\mathrm{N}), 1232(\mathrm{C}=\mathrm{S})$.

2-(4-Methoxybenzylidene)hydrazinecarbothioamide 1i: Yield $88 \%$. mp $172-173{ }^{\circ} \mathrm{C} .{ }^{1} \mathrm{H}-\mathrm{NMR} \delta: 3.80\left(\mathrm{~s}, 3 \mathrm{H}, \mathrm{CH}_{3}\right), 6.96(\mathrm{~d}, 2 \mathrm{H}, J=8.3 \mathrm{~Hz}, \mathrm{Ar}-\mathrm{H})$, $7.76(\mathrm{~d}, 2 \mathrm{H}, J=8.3 \mathrm{~Hz}, \mathrm{Ar}-\mathrm{H}), 7.92$ and $8.12\left(2 \times \mathrm{s}, 2 \mathrm{H},-\mathrm{NH}_{2}\right), 8.03(\mathrm{~s}, 1 \mathrm{H}$, $\mathrm{CH}), 11.33(\mathrm{~s}, 1 \mathrm{H},-\mathrm{NH}-)$. IR $(\mathrm{KBr}) \mathrm{cm}^{-1}: 3390\left(\mathrm{NH}_{2}\right), 3270(\mathrm{NH}), 1610$ $(\mathrm{C}=\mathrm{N}), 1260(\mathrm{C}=\mathrm{S})$.

2-(4-Hydroxy-3-methoxybenzylidene)hydrazinecarbothioamide 1j: Yield $85 \%$. mp $198-199^{\circ} \mathrm{C} .{ }^{1} \mathrm{H}-\mathrm{NMR} \delta: 3.81\left(\mathrm{~s}, 3 \mathrm{H}, \mathrm{CH}_{3}\right), 7.45-7.73(\mathrm{~m}, 3 \mathrm{H}$, Ar-H), 7.90 and $8.09\left(2 \times \mathrm{s}, 2 \mathrm{H},-\mathrm{NH}_{2}\right), 7.93(\mathrm{~s}, 1 \mathrm{H}, \mathrm{CH}), 9.43(\mathrm{~s}, 1 \mathrm{H}, \mathrm{OH})$, $11.24(\mathrm{~s}, 1 \mathrm{H},-\mathrm{NH}-)$. IR (KBr) cm ${ }^{-1}: 3434\left(\mathrm{NH}_{2}\right), 3276(\mathrm{NH}), 1587(\mathrm{C}=\mathrm{N})$, $1276(\mathrm{C}=\mathrm{S})$.

Preparation of 5-Phenyl-4,5-dihydro-1,3,4-thiadiazol Analogues 2a-j A mixture of thiosemicarbazone $(5 \mathrm{mmol})$ and acetic anhydride $(7 \mathrm{ml})$ in ethanol was refluxed with constant stirring for $30 \mathrm{~min}$ and then cooled to ambient temperature. The mixture was poured into ice cold water and neutralized with dilute sodium hydroxide solution. A white precipitate was produced, the mixture was filtered and washed with water to give a crude production. Then it was purified by recrystallization with $\mathrm{N}, \mathrm{N}$-dimethylformamide-EtOH, affording pure thiadiazole derivatives $\mathbf{2} \mathbf{a}-\mathbf{j}$.

$N$-(4-Acetyl-5-phenyl-4,5-dihydro-1,3,4-thiadiazol-2-yl)acetamide 2a: Yield $78 \%$. mp 201-202 ${ }^{\circ} \mathrm{C}$ (lit. ${ }^{13)} \mathrm{mp} 222-223^{\circ} \mathrm{C}$ ). ${ }^{1} \mathrm{H}-\mathrm{NMR} \delta: 2.01$ (s, $\left.3 \mathrm{H},-\mathrm{NHCOCH}_{3}\right), 2.24\left(\mathrm{~s}, 3 \mathrm{H},-\mathrm{COCH}_{3}\right), 6.86(\mathrm{~s}, 1 \mathrm{H}, \mathrm{H}-5), 7.26-7.41(\mathrm{~m}$, $5 \mathrm{H}, \mathrm{Ar}-\mathrm{H}), 11.81$ (s, 1H, -NH-). IR (KBr) cm ${ }^{-1}: 3218,1713,1700,1632$, 1599, 1490. EI-MS m/z (\%): 264 (M $\left.{ }^{+}, 100\right), 222$ (45), 263 (25). Anal. Calcd for $\mathrm{C}_{12} \mathrm{H}_{13} \mathrm{~N}_{3} \mathrm{O}_{2} \mathrm{~S}: \mathrm{C}, 54.74 ; \mathrm{H}, 4.98 ; \mathrm{N}, 15.96$. Found: C, 54.78; H, 4.95; N, 15.98 .

$N$-(4-Acetyl-5- $p$-tolyl-4,5-dihydro-1,3,4-thiadiazol-2-yl)acetamide $\mathbf{2 b}$ : Yield $78 \%$. mp $162-163{ }^{\circ} \mathrm{C} .{ }^{1} \mathrm{H}-\mathrm{NMR} \delta: 2.07$ (s, 3H, $\left.-\mathrm{NHCOCH}_{3}\right), 2.23$ $\left(\mathrm{s}, 3 \mathrm{H},-\mathrm{COCH}_{3}\right), 2.42\left(\mathrm{~s}, 3 \mathrm{H},-\mathrm{CH}_{3}\right), 6.81(\mathrm{~s}, 1 \mathrm{H}, \mathrm{H}-5), 6.81-6.89(\mathrm{~m}, 4 \mathrm{H}$, Ar-H), 11.72 (s, 1H, -NH-). IR ( $\mathrm{KBr}) \mathrm{cm}^{-1}: 3222,1698,1636,1609,1572$, 1490. EI-MS m/z (\%): 278 (M+100), 233 (60), 133 (34). Anal. Calcd for 
$\mathrm{C}_{13} \mathrm{H}_{15} \mathrm{~N}_{3} \mathrm{O}_{2} \mathrm{~S}: \mathrm{C}, 56.30 ; \mathrm{H}, 5.45 ; \mathrm{N}, 15.15$. Found: $\mathrm{C}, 56.33 ; \mathrm{H}, 5.41 ; \mathrm{N}$, 15.19 .

$N$-(4-Acetyl-5-(4-nitrophenyl)-4,5-dihydro-1,3,4-thiadiazol-2-yl)acetamide 2c: Yield $70 \%$. mp $218-219^{\circ} \mathrm{C}$ (lit. ${ }^{13)} \mathrm{mp} 210-211^{\circ} \mathrm{C}$ ). ${ }^{1} \mathrm{H}-$ NMR $\delta: 2.07$ (s, 3H, $\left.-\mathrm{NHCOCH}_{3}\right), 2.28\left(\mathrm{~s}, 3 \mathrm{H},-\mathrm{COCH}_{3}\right), 6.87(\mathrm{~s}, 1 \mathrm{H}, \mathrm{H}-$ 5), 7.54-8.28 (m, 4H, Ar-H), 11.89 (s, $1 \mathrm{H},-\mathrm{NH}-)$. IR (KBr) cm ${ }^{-1}: 3217$, 1697, 1612, 1521, 1446. EI-MS m/z (\%): 309 (M+1 100), 267 (20). Anal. Calcd for $\mathrm{C}_{12} \mathrm{H}_{12} \mathrm{~N}_{4} \mathrm{O}_{4} \mathrm{~S}$ : C, 46.75; H, 3.92; N, 18.17. Found: C, 46.78; H, $3.91 ; \mathrm{N}, 18.20$.

$N$-(4-Acetyl-5-(3-nitrophenyl)-4,5-dihydro-1,3,4-thiadiazol-2-yl)acetamide 2d: Yield 67\%. mp 233-234 ${ }^{\circ} \mathrm{C} .{ }^{1} \mathrm{H}-\mathrm{NMR} \delta: 2.08(\mathrm{~s}, 3 \mathrm{H}$, $\left.-\mathrm{NHCOCH}_{3}\right), 2.26\left(\mathrm{~s}, 3 \mathrm{H},-\mathrm{COCH}_{3}\right), 6.82(\mathrm{~s}, 1 \mathrm{H}, \mathrm{H}-5), 7.69-8.21(\mathrm{~m}, 4 \mathrm{H}$, $\mathrm{Ar}-\mathrm{H}), 11.78$ (s, 1H, -NH-). IR (KBr) cm ${ }^{-1}: 3222,1695,1631,1601,1526$, 1489. EI-MS m/z (\%): $309\left(\mathrm{M}^{+}, 85\right), 267$ (100). Anal. Calcd for $\mathrm{C}_{12} \mathrm{H}_{12} \mathrm{~N}_{4} \mathrm{O}_{4} \mathrm{~S}: \mathrm{C}, 46.75 ; \mathrm{H}, 3.92 ; \mathrm{N}, 18.17$. Found: $\mathrm{C}, 46.79 ; \mathrm{H}, 3.89 ; \mathrm{N}$, 18.15 .

$\mathrm{N}$-(4-Acetyl-5-(3-(dimethylamino)phenyl)-4,5-dihydro-1,3,4-thiadiazol-2yl)acetamide 2e: Yield $70 \%$. mp $239-240{ }^{\circ} \mathrm{C}$ (lit. ${ }^{13)} \mathrm{mp} 222^{\circ} \mathrm{C}$ ). ${ }^{1} \mathrm{H}-\mathrm{NMR}$ $\delta: 2.07\left(\mathrm{~s}, 3 \mathrm{H},-\mathrm{NHCOCH}_{3}\right), 2.19\left(\mathrm{~s}, 3 \mathrm{H},-\mathrm{COCH}_{3}\right), 2.90(\mathrm{~s}, 6 \mathrm{H}$, $\left.-\mathrm{N}\left(\mathrm{CH}_{3}\right)_{2}\right), 6.68-7.70(\mathrm{~m}, 2 \mathrm{H}, \mathrm{Ar}-\mathrm{H}), 6.75(\mathrm{~s}, 1 \mathrm{H}, \mathrm{H}-5), 7.08-7.10(\mathrm{~m}$, $2 \mathrm{H}, \mathrm{Ar}-\mathrm{H}), 11.73$ (s, 1H, -NH-). IR (KBr) cm $\mathrm{cm}^{-1}: 3207,1695,1633,1608$, 1519, 1485. EI-MS m/z (\%): $307\left(\mathrm{M}^{+}, 100\right), 265$ (30). Anal. Calcd for $\mathrm{C}_{14} \mathrm{H}_{18} \mathrm{~N}_{4} \mathrm{O}_{2} \mathrm{~S}:$ C, 54.88; H, 5.92; N, 18.29. Found: C, 54.85; H, 5.94; N, 18.26 .

$\mathrm{N}$-(4-Acetyl-5-(4-chlorophenyl)-4,5-dihydro-1,3,4-thiadiazol-2-yl)acetamide 2f: Yield $73 \%$. mp $233-234{ }^{\circ} \mathrm{C}$ (lit. ${ }^{13)} \mathrm{mp} 229-233^{\circ} \mathrm{C}$ ). ${ }^{1} \mathrm{H}-$ NMR $\delta: 2.12\left(\mathrm{~s}, 3 \mathrm{H},-\mathrm{NHCOCH}_{3}\right), 2.23\left(\mathrm{~s}, 3 \mathrm{H},-\mathrm{COCH}_{3}\right), 6.88(\mathrm{~s}, 1 \mathrm{H}, \mathrm{H}-5)$ $7.29-7.47(\mathrm{~m}, 4 \mathrm{H}, \mathrm{Ar}-\mathrm{H}), 11.85(\mathrm{~s}, 1 \mathrm{H},-\mathrm{NH}-) . \mathrm{IR}(\mathrm{KBr}) \mathrm{cm}^{-1}: 3215$, 1701, 1637, 1612, 1542, 1492. EI-MS $m / z$ (\%): $299(\mathrm{M}+2,39), 297\left(\mathrm{M}^{+}\right.$, 100), 252 (80), 144 (20). Anal. Calcd for $\mathrm{C}_{12} \mathrm{H}_{12} \mathrm{ClN}_{3} \mathrm{O}_{2} \mathrm{~S}: \mathrm{C}, 48.40 ; \mathrm{H}, 4.06$; N, 14.11. Found: C, $48.45 ; \mathrm{H}, 4.02 ; \mathrm{N}, 14.14$.

$N$-(4-Acetyl-5-(4-bromophenyl)-4,5-dihydro-1,3,4-thiadiazol-2-yl)acetamide 2g: Yield $78 \%$. mp $132-133{ }^{\circ} \mathrm{C} .{ }^{1} \mathrm{H}-\mathrm{NMR} \quad \delta: 2.10(\mathrm{~s}, 3 \mathrm{H}$, $\left.-\mathrm{NHCOCH}_{3}\right), 2.25\left(\mathrm{~s}, 3 \mathrm{H},-\mathrm{COCH}_{3}\right), 6.89(\mathrm{~s}, 1 \mathrm{H}, \mathrm{H}-5)$ 7.30-7.49 (m, 4H, $\mathrm{Ar}-\mathrm{H}), 11.78$ (s, 1H, -NH-). IR (KBr) cm ${ }^{-1}: 3215,1699,1631,1612,1542$, 1492. EI-MS $m / z$ (\%): $344(\mathrm{M}+3,37), 343(\mathrm{M}+2,100), 342(\mathrm{M}+1,35)$, $341\left(\mathrm{M}^{+}, 98\right), 296$ (77), 188 (25). Anal. Calcd for $\mathrm{C}_{12} \mathrm{H}_{12} \mathrm{BrN}_{3} \mathrm{O}_{2} \mathrm{~S}: \mathrm{C}, 42.12$; H, 3.53; N, 12.28. Found: C, 42.16; H, 3.51; N, 12.31 .

$N$-(4-Acetyl-5-(4-hydroxyphenyl)-4,5-dihydro-1,3,4-thiadiazol-2-yl)acetamide $2 \mathrm{~h}$ : Yield $80 \%$. mp $210-211^{\circ} \mathrm{C}$. ${ }^{1} \mathrm{H}-\mathrm{NMR} \quad \delta: 2.06(\mathrm{~s}, 3 \mathrm{H}$, $\left.-\mathrm{NHCOCH}_{3}\right), 2.23\left(\mathrm{~s}, 3 \mathrm{H},-\mathrm{COCH}_{3}\right), 6.81(\mathrm{~s}, 1 \mathrm{H}, \mathrm{H}-5), 6.91-6.99(\mathrm{~m}, 4 \mathrm{H}$, $\mathrm{Ar}-\mathrm{H}), 11.63$ (s, 1H, -NH-). IR (KBr) cm ${ }^{-1}: 3217,1712,1701,1633,1608$, 1454. EI-MS $m / z(\%): 280\left(\mathrm{M}^{+}, 100\right), 256$ (30). Anal. Calcd for $\mathrm{C}_{12} \mathrm{H}_{13} \mathrm{~N}_{3} \mathrm{O}_{3} \mathrm{~S}$ : C, 51.60; H, 4.69; N, 15.04. Found: C, 51.64; H, 4.67; N, 15.08 .

$\mathrm{N}$-(4-Acetyl-5-(4-methoxyphenyl)-4,5-dihydro-1,3,4-thiadiazol-2-yl)acetamide 2i: Yield $78 \%$. mp $156-158^{\circ} \mathrm{C}$ (lit. ${ }^{13)} \mathrm{mp} 168-169^{\circ} \mathrm{C}$ ). ${ }^{1} \mathrm{H}-$ NMR $\delta: 2.07\left(\mathrm{~s}, 3 \mathrm{H},-\mathrm{NHCOCH}_{3}\right), 2.24\left(\mathrm{~s}, 3 \mathrm{H},-\mathrm{COCH}_{3}\right), 3.77(\mathrm{~s}, 3 \mathrm{H}$, $\left.-\mathrm{OCH}_{3}\right), 6.81$ (s, 1H, H-5), 6.96-7.16 (m, 4H, Ar-H), $11.72(\mathrm{~s}, 1 \mathrm{H},-\mathrm{NH}-)$. IR (KBr) cm ${ }^{-1}: 3222,1698,1636,1609,1572,1490$. EI-MS $m / z$ (\%): 294 $\left(\mathrm{M}^{+}, 100\right), 252(80), 144(20)$. Anal. Calcd for $\mathrm{C}_{13} \mathrm{H}_{15} \mathrm{~N}_{3} \mathrm{O}_{3} \mathrm{~S}: \mathrm{C}, 53.23 ; \mathrm{H}$, $5.15 ; \mathrm{N}, 14.32$. Found: C, 53.25; H, 5.11; N, 14.35 .

$N$-(4-Acetyl-5-(4-hydroxy-3-methoxyphenyl)-4,5-dihydro-1,3,4-thiadiazol-2-yl)acetamide 2j: Yield $67 \%$. mp $228-230{ }^{\circ} \mathrm{C} .{ }^{1} \mathrm{H}-\mathrm{NMR} \delta: 2.02$ (s, $\left.3 \mathrm{H},-\mathrm{NHCOCH}_{3}\right), 2.30\left(\mathrm{~s}, 3 \mathrm{H},-\mathrm{COCH}_{3}\right), 3.84\left(\mathrm{~s}, 3 \mathrm{H},-\mathrm{OCH}_{3}\right), 6.89(\mathrm{~s}, 1 \mathrm{H}$, C5-H), 7.11-7.47 (m, 3H, Ar-H), $9.13(\mathrm{~s}, 1 \mathrm{H},-\mathrm{OH}), 11.82(\mathrm{~s}, 1 \mathrm{H},-\mathrm{NH}-)$. IR (KBr) cm ${ }^{-1}: 3203,1704,1639,1605,1496,1441$. EI-MS $m / z(\%): 310$ ( $\left.\mathrm{M}^{+}, 100\right), 266$ (45), 214 (20). Anal. Caled for $\mathrm{C}_{13} \mathrm{H}_{15} \mathrm{~N}_{3} \mathrm{O}_{4} \mathrm{~S}$ : C, 50.47; H, $4.89 ; \mathrm{N}, 13.58$. Found: C, 50.53; H, 4.85; N, 13.62 .

Cytotoxicity Assay Cytotoxicity after treatment of the tumor cells with the test materials was determined using the SRB (sulforhodamine-B) method, currently adopted in the NCI's in vitro anti-cancer drug screening, ${ }^{18)}$ i.e., the inhibition rate of cell proliferation was estimated after continuous exposure to the test materials for $48 \mathrm{~h}$. All samples were tested in triplicate and the mean $\mathrm{IC}_{50}$ values $(\mu \mathrm{g} / \mathrm{ml})$ (the concentration of the compound that resulted in a 50\% inhibition of cell proliferation) and the S.E.M. were calculated, respectively.

\section{References}

1) Demirbas A., Sahin D., Demirbas N., Karaoglu S. A., Eur. J. Med. Chem., 44, 2896-2903 (2009).

2) Kumar D., Maruthi Kumar N., Chang K.-H., Shah K., Eur. J. Med. Chem., 45, 4664-4668 (2010).

3) Foroumadi A., Kargar Z., Sakhteman A., Sharifzadeh Z., Feyzmohammadi R., Kazemi M., Shafiee A., Bioorg. Med. Chem. Lett., 16, 1164-1167 (2006).

4) Michael R., Stillings M. R., Welbourn A. P., Walter D. S., J. Med. Chem., 29, 2280-2284 (1986).

5) Schenone S. S., Brullo C. C., Bruno O. O., Bondavalli F. F., Ranise A. A., Filippelli W. W., Rinaldi B. B., Capuano A. A., Falcone G. G., Bioorg. Med. Chem., 14, 1698-1705 (2006).

6) Clerici F., Pocar D., Guido M., Loche A., Perlini V., Brufani M., J. Med. Chem., 44, 931-936 (2001).

7) Chen Z., Xu W., Liu K., Yang S., Fan H., Bhadury P. S., Hu D.-Y., Zhang Y., Molecules, 15, 9046-9056 (2010).

8) Stockler M. R., Wilcken N. J. C., Coates A. S., Breast Cancer Res. Treat., 81, 49-52 (2003).

9) Foroumadi A., Soltani F., Moallemzadeh-Haghighi H., Shafiee A., Arch. Pharm. (Weinheim), 338, 112-116 (2005).

10) Senff-Ribeiro A., Echevarria A., Silva E. F., Veiga S. S., Oliveira M. B., Anticancer Drugs, 15, 269-275 (2004).

11) Asbury R., Blessing J. A., Moore D., Am. J. Clin. Oncol., 19, 400402 (1996)

12) Engstrom P. F., Ryan L. M., Falkson G., Haller D. G., Am. J. Clin. Oncol., 14, 33-35 (1991).

13) Kubota S., Ueda Y., Fujikane K., Toyooka F., Shibuya M., J. Org. Chem., 45, 1473-1477 (1980).

14) Toyooka K., Kasai T., Hori S., Kawashima Y., Shibuya M., Kubota S., Chem. Pharm. Bull., 39, 2837-2841 (1991).

15) Askari S. H., Moss S. F., Taylor D. R., J. Chem. Soc., Perkin Trans. 1, 1981, 360-365 (1981).

16) Janos E., Oros G., Pharmacochemistry Library, 16, 385-388 (1991).

17) Alam M. S., Liu L., Lee Y. E., Lee D. U., Chem. Pharm. Bull., 59, 568-573 (2011).

18) Skehan P., Streng R., Scudiero D., Monks A., McMahon J., Vistica D., Warren J. T., Bokesch H., Kenney S., Boyd M. R., J. Natl. Cancer Inst., 82, 1107-1112 (1990). 